\title{
Novel $\mathbf{N}-1$ Substituted Fluoroquinolones Inhibit Human Topoisomerase I Activity and Exhibit Anti-proliferative Activity
}

\author{
Lisa M. Oppegard ${ }^{\# 1,5}$, Justine L. Delgado\#2, Chaitanya A. Kulkarni' ${ }^{2}$, Tyrell R. Towle ${ }^{2,6}$, \\ Delaney E. Hart ${ }^{1}$, Bridget P. Williams ${ }^{1}$, Sarah R.C. Lentz ${ }^{1}$, Beverly J. Norris ${ }^{3}$, Craig M. \\ Flory $^{3}$, Robert J. Schumacher ${ }^{3}$, Daryl J. Murry ${ }^{4,7}$, Robert J. Kerns ${ }^{2}$, and Hiroshi Hiasa ${ }^{1,{ }^{*}}$ \\ ${ }^{1}$ Department of Pharmacology, University of Minnesota Medical School, 6-120 Jackson Hall, 321 \\ Church Street SE, Minneapolis, Minnesota 55455, U.S.A. \\ 2Division of Medicinal and Natural Products Chemistry, Department of Pharmaceutical Sciences \\ and Experimental Therapeutics, College of Pharmacy, University of lowa, 115 S Grand Ave., \\ S321 Pharmacy Building, lowa City, lowa 52242, U.S.A. \\ ${ }^{3}$ Center for Translational Medicine, University of Minnesota Academic Health Center, 515 \\ Delaware Street SE, Room 2-191, Minneapolis, Minnesota 55455, U.S.A. \\ 4The Division of Pharmaceutics and Translational Therapeutics, Department of Pharmaceutical \\ Sciences and Experimental Therapeutics, College of Pharmacy, University of lowa, $115 \mathrm{~S}$ Grand \\ Ave., S321 Pharmacy Building, lowa City, lowa 52242, U.S.A. \\ \# These authors contributed equally to this work.
}

\begin{abstract}
Fluoroquinolone-class agents selectively target the bacterial type IIA topoisomerases DNA gyrase and topoisomerase IV, with a few exceptions that target eukaryotic type IIA topoisomerases. Fluoroquinolones bind and stabilize type IIA topoisomerase-DNA covalent complexes that contain a double-strand break. This unique mode of action is referred to as 'topoisomerase poisoning'. We discovered that two novel fluoroquinolones having aryl functionality at the $\mathrm{N}-1$ position, UITT-3217 (217) and UITT-3-227 (227), could inhibit the catalytic activity of human topoisomerase II without stabilizing topoisomerase-DNA complexes, i.e., without poisoning it. Surprisingly, these compounds are more effective in inhibiting the catalytic activities of human and bacterial topoisomerase I. The National Cancer Institute's 60 human tumor cell lines screen revealed significant anti-proliferative activities with 217 and 227 against the majority of 60 cancer cell lines. A proof of concept in vivo efficacy study using an HT-29 xenograft model of human
\end{abstract}

\footnotetext{
${ }^{*}$ Corresponding author. Tel. 612-626-3101, hiasa001@umn.edu.

${ }^{5}$ College of Undergraduate Health Sciences, Northwestern Health Sciences University, Bloomington, Minnesota 55431, U.S.A.

6 MedPharm Holdings, Denver, Colorado 80239, U.S.A.

${ }^{7}$ Department of Pharmacy Practice, College of Pharmacy, University of Nebraska Medical Center, Omaha, Nebraska 68198, U.S.A. Conflict of Interest

The authors declare no competing financial interest.

Ethical approval

This article does not contain any studies with human participants performed by any of the authors. The animal studies were approved by the University of Minnesota Institutional Animal Care and Use Committee.

Informed consent

For this type of study, formal consent is not required.
} 
colorectal cancer showed that 217 could inhibit the proliferation of human colorectal cancer cells to a degree comparable to fluorouracil in mice. Although 227 also exhibited anti-proliferative activity, it was not as effective as 217 in this xenograft model. These novel fluoroquinolones may serve as promising lead compounds for the development of new anticancer drugs.

\section{Keywords}

colon cancer; DNA intercalator; fluoroquinolone; topoisomerase I; topoisomerase II

\section{Introduction}

DNA topoisomerases are responsible for controlling the topology of DNA (1-3). Human cells contain three types of topoisomerases, type IA, type IB, and type IIA. Each class of topoisomerases is structurally and biochemically conserved and forms a large protein family (1-3). Type IA topoisomerases consist of topoisomerase III (Topo III), including the two isoforms of human Topo III, and bacterial topoisomerase I (Topo I). Nuclear Topo I (hTopo I) and mitochondrial Topo I in human cells are type IB topoisomerases. All type I topoisomerases cleave one strand of duplex DNA, pass the other strand through the nick, and then reseal the broken strand. Type IA topoisomerases covalently attach the active-site tyrosine to a 5 '-phosphoryl group and utilize the 'strand passage' mechanism to relax negative supercoils, whereas type IB topoisomerases covalently attach the active-site tyrosine to a 3 '-phosphoryl group and utilize the 'swivel' mechanism to relax negative and positive supercoils (1-3). Type IIA topoisomerases include the two isoforms of human topoisomerase II (hTopo II), as well as bacterial DNA gyrase and topoisomerase IV. Type IIA topoisomerases cleave both strands of duplex DNA, pass another segment of duplex DNA through the double-strand break (DSB), and then reseal the broken strands. Type IIA topoisomerases form phosphotyrosyl bonds between the two active-site tyrosines and a pair of $5^{\prime}$-phosphates, and utilize the 'strand passage' mechanism to alter the topology of DNA $(1-3)$.

In recent years, many cancer-specific anticancer agents have been developed and significant advances have been made toward precision medicine in cancer treatment (4). However, traditional or non-specific anticancer drugs are still important for the treatment of patients whose cancers either do not respond or have developed resistance to cancer-specific anticancer agents. Thus, there is a need to develop novel anticancer agents. Topoisomerases, especially type IIA topoisomerases, are proven therapeutic targets of anticancer and antibacterial drugs (2,5-7). Clinically successful hTopo II inhibitors, such as etoposide, trap hTopo II-DNA covalent complexes as hTopo II-drug-DNA ternary complexes. This leads to an increase in the level of topoisomerase-catalyzed DSB formation in cells. This unique mode of action is often referred to as 'topoisomerase poisoning'. Topoisomerase poisoning is also utilized by antibacterial drugs that target bacterial type IIA topoisomerases, such as fluoroquinolones, and anticancer drugs that target hTopo I, such as irinotecan (2,5-7). Topoisomerase poisoning is an effective cell-killing mechanism but it also causes genotoxicities, including the possibility of therapy-related acute myeloid leukemia (8). Thus, there is an unmet need to identify new topoisomerase inhibitors that act through a 
mechanism different from topoisomerase poisoning, which could allow for the development of novel anticancer agents with improved efficacy and/or superior safety profiles. Drugs with novel chemical structures and/or new mechanisms might also be effective against the cancers that are resistant to current topoisomerase-targeting anticancer drugs.

Fluoroquinolones are best known as selective inhibitors of bacterial type IIA topoisomerases, although some fluoroquinolones, such as CP-115953, have been reported to poison hTopo II (9). During our studies on novel fluoroquinolones with extended aryl functionality at the $N-1$ position, we discovered that N1-biphenyl fluoroquinolone UITT-3217 (217) and N1-napthyl fluoroquinolone UITT-3-227 (227) could inhibit the catalytic activity of hTopo II without poisoning it (10). Here, we further assessed the activities of 217 and 227. Surprisingly, these fluoroquinolones inhibited the catalytic activities of both eukaryotic and bacterial Topo Is. In fact, they inhibited hTopo I activity more effectively than hTopo II activity, and acted as a catalytic inhibitor, not poison, of hTopo I. Furthermore, these compounds exhibited significant anti-proliferative activities both in vitro and in vivo. Therefore, 217 and 227 represent novel chemical structures that inhibit both hTopo I and hTopo II through a mechanism different from topoisomerase poisoning, and may serve as promising lead compounds for the development of a novel class of anticancer drug.

\section{Methods}

\section{Synthesis of N-1 fluoroquinolones}

Synthesis of 217 and 227 is described by Towle et al. (10). The modifications made for a large-scale synthesis are described in detail in the Supplemental Data.

\section{Relaxation assay for eukaryotic Topo I}

Calf Topo I (cTopo I) purchased from Thermo Fisher Scientific and hTopo I purchased from Topogen were used in this study. One unit of Topo I was defined as the amount of topoisomerase required to completely relax $0.3 \mu \mathrm{g}$ of the negatively-supercoiled pBR322 plasmid DNA under the conditions described below.

Relaxation reaction mixtures $(20 \mu \mathrm{L})$ contained $50 \mathrm{mM}$ Tris- $\mathrm{HCl}\left(\mathrm{pH} 7.5\right.$ at $\left.23^{\circ} \mathrm{C}\right), 100 \mathrm{mM}$ $\mathrm{NaCl}, 10 \mathrm{mM} \mathrm{MgCl} 2,1 \mathrm{mM}$ dithiothreitol (DTT), $50 \mu \mathrm{g} / \mathrm{ml}$ bovine serum albumin (BSA), $0.3 \mu \mathrm{g}$ of the supercoiled plasmid DNA, 1 unit of either human or calf Topo I, and the various concentrations of fluoroquinolones (11). Reaction mixtures were incubated at $37^{\circ} \mathrm{C}$ for 15 minutes and terminated by adding ethylenediaminetetraacetic acid (EDTA) to $25 \mathrm{mM}$. The DNA products were analyzed by electrophoresis through vertical $1.2 \%$ agarose gels at 2 $\mathrm{V} / \mathrm{cm}$ for 15 hours in TAE buffer. Gels were stained with $0.5 \mu \mathrm{g} / \mathrm{ml}$ ethidium bromide, and then photographed and quantified using a MyECL Imager (Thermo Fisher Scientific).

\section{DNA cleavage assay for eukaryotic Topo I}

DNA cleavage reaction mixtures $(20 \mu \mathrm{l})$ contained $50 \mathrm{mM}$ Tris- $\mathrm{HCl}\left(\mathrm{pH} 7.5\right.$ at $\left.23^{\circ} \mathrm{C}\right), 10$ $\mathrm{mM} \mathrm{MgCl} 2,1 \mathrm{mM}$ DTT, $50 \mu \mathrm{g} / \mathrm{ml} \mathrm{BSA}, 0.3 \mu \mathrm{g}$ of the supercoiled plasmid DNA, 10 units of either human or calf Topo I, and the various concentrations of fluoroquinolones (11). Reaction mixtures were incubated at $37^{\circ} \mathrm{C}$ for 15 minutes. Sodium dodecyl sulfate (SDS) 
was added to a concentration of $1 \%$, and the reaction mixtures were further incubated at $37^{\circ} \mathrm{C}$ for 5 minutes. EDTA and proteinase $\mathrm{K}$ were then added to $25 \mathrm{mM}$ and $100 \mu \mathrm{g} / \mathrm{mL}$, respectively, and the incubation was continued for 30 minutes at $37^{\circ} \mathrm{C}$. The DNA products were purified by extraction with phenol/chloroform/isoamyl alcohol (25:24:1, v/v) and then analyzed by electrophoresis through vertical $1.2 \%$ agarose gels at $2 \mathrm{~V} / \mathrm{cm}$ for 15 hours in TAE buffer that contained $0.5 \mu \mathrm{g} / \mathrm{mL}$ ethidium bromide. After destaining in water, gels were photographed and quantified using a MyECL Imager.

\section{The 60 human tumor cell lines screening}

The 60 human tumor cell lines screening was performed at the National Cancer Institute (NCI). Both one-dose and five-dose screenings were performed with 217 and 227 using the protocol and 60 human cancer cell lines established at the NCI (19). The $\mathrm{GI}_{50}$ value is the concentration of a compound resulting in a 50\% reduction in the net protein increase in control cells during the drug incubation. The $\mathrm{LC}_{50}$ value is the concentration of a compound resulting in a 50\% reduction in the measured protein at the end of the drug treatment as compared to that at the beginning (19).

\section{In vivo efficacy study}

Forty female athymic nude, outbred mice (CRL strain code 490) at approximately 6-8 weeks old were used [n=10 per group for 4 test groups; vehicle, 217, 227, or fluorouracil (5FU)]. HT-29 human colon carcinoma cells in log phase growth were injected subcutaneously into the right flank in a solution of cell culture medium without FBS and with 40-50\% matrigel at a concentration of 0.5 to $1 \times 10^{6}$ cells in a $100 \mu \mathrm{L}$ volume; tumors grew in all mice implanted with cells. Six days post cell injection (day 0) individual mice were assigned to groups to balance tumor volume among groups, and then groups were randomized to treatment assignment (restricted randomization). 217 and 227 stock solutions were made in sterile DMSO, at a concentration of $50 \mathrm{mg} / \mathrm{ml}$, and stored at $4^{\circ} \mathrm{C}$. 217 and 227 for intraperitoneal (IP) administration were formulated in sterile USP water (with a final concentration of 4-6\% DMSO), and the $\mathrm{pH}$ of the solution was recorded for each batch of prepared drug. The dose level was $30 \mathrm{mg} / \mathrm{kg}$ daily, a tolerated dose of these compounds for repeat administration. The volume administered was 200 to $300 \mu \mathrm{L}$ per dose, once daily. Dosing was initiated one day post-randomization (day 1). 5-FU for IP administration was formulated in sterile USP water. It is supplied as an aqueous solution intended for dilution with a suitable parenteral fluid prior to intravenous infusion. The volume administered was 200 to $300 \mu \mathrm{L}$ per dose. Three doses of $60 \mathrm{mg} / \mathrm{kg}$ were administered at a frequency of once every 7 days (days 1, 8 and 15 of study). The animal studies were approved by the University of Minnesota Institutional Animal Care and Use Committee.

\section{Results and Discussion}

The structures of topoisomerase-fluoroquinolone-DNA ternary complexes have shown that the $N-1$ substituent on the quinolone core is located near the active site tyrosine, and a hydrophobic pocket of the topoisomerase (12-14). As part of our efforts to create new fluoroquinolones with improved potency, we designed and synthesized a panel of novel fluoroquinolones with extended alkyl and aryl functionality at the $N-1$ position in an effort 
to create an additional fluoroquinolone-topoisomerase contact in ternary complexes (10). During the initial characterization of these novel fluoroquinolones, we discovered that 217 and 227 could inhibit the catalytic activity of the a isoform of hTopo II ( $\mathrm{IC}_{50}$ values for 217 and 227 in a decatenation assay were $127.6 \mu \mathrm{M}$ and $164.6 \mu \mathrm{M}$, respectively) without poisoning it (10). Although their activities were modest, we decided to further examine the activities of these compounds because of the potential impact of discovering novel inhibitors of hTopo II that did not generate DSBs.

\section{7 and 227 inhibit the catalytic activity of type I topoisomerases}

In our attempt to perform a DNA unwinding assay (15) to determine if 217 and 227 intercalate into DNA, we fortuitously discovered that 217 and 227 inhibited the relaxation activity of cTopo I (Fig. S1). Because these results were unexpected, we further assessed the activities of 217 and 227 against other type I topoisomerases. Relaxation assays with either human or Escherichia coli (E. coli) Topo I showed that both 217 and 227 could also inhibit the catalytic activities of these type I topoisomerases (Table 1). In fact, both 217 and 227 inhibited hTopo I activity to a greater extent than hTopo II activity. DNA cleavage assays for eukaryotic (type IB) Topo I, where the poisoning of Topo I would result in an increase in the amount of the nicked DNA, showed that 217 and 227 did not poison either calf or human Topo I (Fig. 1). These results showed 217 and 227 were more effective catalytic inhibitors of type IB topoisomerases than type IIA topoisomerases.

Fluoroquinolones are known to intercalate into DNA (16). However, because 217 and 227 inhibited eukaryotic Topo I, we could not conduct a DNA unwinding assay to determine if these fluoroquinolones also intercalate into DNA. Thus, a thiazole orange displacement assay (17) was employed to examine if they can intercalate into DNA. Both 217 and 227 could displace thiazole orange, suggesting that they intercalate into DNA (Fig. S2). In fact, these fluoroquinolones displaced thiazole orange more efficiently than control fluoroquinolones UING-5-249 (18) and moxifloxacin. These results demonstrated that both 217 and 227 were capable of binding to DNA in the absence of any topoisomerase. Therefore, 217 and 227 may intercalate into DNA and interfere with the catalytic activities of topoisomerases.

Because 217 and 227, which have an extended aryl group at the N1 position, inhibit hTopo I through a mechanism different from that used by typical fluoroquinolones, it is possible that changes to the quinolone core structure can be made. Studies are underway to determine the structural requirements for this novel class of N1-substituted fluoroquinolone to inhibit catalytic activity of hTopo I.

\section{7 and 227 exhibit anti-proliferative activities in vitro and in vivo}

Although the inhibitory effects of 217 and 227 on the catalytic activity of both hTopo I and hTopo II were modest, we considered the possibility that dual inhibition of hTopo I and hTopo II might produce enough of a synergistic effect to more potently inhibit the proliferation of cancer cells. To test this possibility, 217 and 227 were submitted to the NCI for the 60 DTP Human Tumor Cell Line Screen to determine their anti-proliferative activities in vitro (19). The initial single-dose $(10 \mu \mathrm{M})$ screening showed significant growth 
inhibition by both 217 and 227 and further evaluation of their activities determined the mean $\mathrm{GI}_{50}$ values of 217 and 227 to be $1.9 \mu \mathrm{M}$ and $3.1 \mu \mathrm{M}$, respectively, against the 60 cancer cell lines (Table 2). COMPARE analysis (NCI) showed that the $\mathrm{GI}_{50}$ values of both 217 and 227 are significantly lower than that of etoposide, a hTopo II poison used in the treatment of variety of cancers (Fig. S3). Thus, 217 and 227 exhibited significant in vitro antiproliferative activity against many human cancer cell lines.

The in vitro anti-proliferative activity of either 217 or 227 may be solely due to the catalytic inhibition of hTopo I, but it is possible that a synergy may be produced through dual inhibition of hTopo I and hTopo II. We cannot exclude the possibility that these compounds inhibit the activity of additional targets, including mitochondrial Topo I and/or human Topo III, that have not been identified.

Because 217 and 227 exhibited significant anti-proliferative activities in vitro, we conducted a proof of concept efficacy study in vivo. First, we performed in vivo toxicity studies to establish the maximum tolerated doses of 217 and 227 in mice. Daily, IP administration of 217 and 227 at up to $30 \mathrm{mg} / \mathrm{kg}$ for 3 weeks was well-tolerated and did not have any significant effect on animal weight gain (Fig. S4). Higher doses could not be used due to the limited aqueous solubility of these compounds. Based on the in vitro susceptibility to 217 and 227 (data not shown) and the availability of the established xenograft models, we used a human colon cancer (HT-29) xenograft model for the initial in vivo efficacy study. Since 5$\mathrm{FU}$ is one of the first line drugs used for colon cancer treatment (20), 5-FU was used as a control drug. 217 inhibited the proliferation of colon cancer in mice comparable to the effect of 5-FU (Fig. 2). 227 exhibited some activity but was not as effective as 217 in this xenograft model.

217 and 227 represent novel chemical structures and new mechanism to inhibit human topoisomerases. Successful translation of these compounds and/or their analogs may lead to the development of novel anticancer agents.

\section{Supplementary Material}

Refer to Web version on PubMed Central for supplementary material.

\section{Acknowledgements}

We would like to thank Margaret A. Mysz, Lia G. Coicou, Brenda L. Koniar, and Nicole A. Larson for expert assistance.

Funding

This work was supported in part by the National Institutes of Health (NIH) grant R01 AI087671 (to RJK), the NIH's National Center for Advancing Translational Sciences grant UL1TR002494 (to HH), and the National Cancer Institute's Developmental Therapeutic Program. We also received support from the University of Minnesota's Center for Translational Medicine and the Masonic Cancer Center (to RJS). TRT acknowledges support of the NIH Predoctoral Training Program in Pharmacological Sciences (GM067795), the American Foundation for Pharmaceutical Education Predoctoral Fellowship Program, and the American Chemical Society Division of Medicinal Chemistry Fellowship sponsored by Richard B. Silverman-2012 Predoctoral. CAK and JLD acknowledge support of training fellowships from the University of Iowa Center for Biocatalysis and Bioprocessing and of the NIH-sponsored Predoctoral Training Program in Biotechnology (GM008365). The content of this article is solely the responsibility of the authors and does not necessarily represent the official views of the National Center for Advancing Translational Sciences. 


\section{References}

1. Corbett KD, Berger JM (2004) Structure, molecular mechanisms, and evolutionary relationships in DNA topoisomerases. Annu Rev Biophys Biomol Struc 33:95-118

2. Chen SH, Chan NL, Hsieh TS (2013) New mechanistic and functional insights into DNA topoisomerases. Annu Rev Biochem 82:139-170 [PubMed: 23495937]

3. Pommier Y, Sun Y, Huang SN, Nitiss JL (2016) Roles of eukaryotic topoisomerases in transcription, replication and genomic stability. Nat Rev Mol Cell Biol 17:703-721. [PubMed: 27649880]

4. Precision Medicine. https://www.cancer.gov/about-cancer/treatment/types/precision-medicine. Accessed August 2, 2018

5. Nitiss JL (2009) Targeting DNA topoisomerase II in cancer chemotherapy. Nat Rev Cancer 9:338350 [PubMed: 19377506]

6. Pommier Y (2013) Drugging topoisomerases: lessons and challenges. ACS Chem Biol 8:82-95 [PubMed: 23259582]

7. Delgado JL, Hsieh CM, Chan NL, Hiasa(2018) Topoisomerases as anticancer targets. Biochem J 475:373-398 [PubMed: 29363591]

8. Pendleton MJ, Lindsey RH, Jr, Felix CA, Grimwade D, Osheroff N (2014) Topoisomerase II and leukemia. Ann NY Acad Sci 1310:98-110 [PubMed: 24495080]

9. Robinson MJ, Martin BA, Gootz TD, McGuirk PR, Moynihan M, Sutcliffe JA, Osheroff N (1991) Effects of quinolone derivatives on eukaryotic topoisomerase II. A novel mechanism for enhancement of enzyme-mediated DNA cleavage. J Biol Chem 266:14585-14592 [PubMed: 1650363]

10. Towle TR, Kulkami CA, Oppegard LM, Williams BP, Picha TA, Hiasa H, Kerns RJ (2018) Design, synthesis, and evaluation of novel $N$-1 fluoroquinolone derivatives: probing for binding contact with the active site tyrosine of gyrase. Bioorg Med Chem Lett 28:1903-1910 [PubMed: 29661533]

11. Oppegard LM, Nguyen T, Ellis KC, Hiasa H (2012) Inhibition of Human Topoisomerases I and II by Simocyclinone D8. J Nat Prod 75:485-489

12. Wohlkonig A, Chan PF, Fosberry AP, Homes P, Huang J, Kranz M, Leydon VR, Miles TJ, Pearson ND, Perera RL, Shillings AJ, Gwynn MN, Bax BD (2010) Structural basis of quinolone inhibition of type IIA topoisomerases and target-mediated resistance. Nat Struct Mol Biol 17:1152-1153 [PubMed: 20802486]

13. Laponogov I, Pan XS, Veselkov DA, McAuley KE, Fisher LM. Sanderson MR(2010) Structural basis of gate-DNA breakage and resealing by type II topoisomerases. PLoS One 5:e11338 [PubMed: 20596531]

14. Blower TR, Williamson BH, Kerns RJ, Berger JM (2016) Crystal structure and stability of gyrasefluoroquinolone cleaved complexes from Mycobacterium tuberculosis. Proc Natl Acad Sci USA 113:1706-1713 [PubMed: 26792525]

15. Fortune JM, Osheroff N (1998) Merbarone inhibits the catalytic activity of human topoisomerase IIa by blocking DNA cleavage. J Biol Chem 273: 17643-17650 [PubMed: 9651360]

16. Palu G, Valisena S, Ciarrochi G, Gatto B, Palumbo M (1992) Quinolone binding to DNA is mediated by magnesium ions. Proc Natl Acad Sci USA 89:9671-9675 [PubMed: 1409681]

17. Boger DL, Tse WC (2001) Thiazole orange as the fluorescent intercalator in a high resolution fid assay for determining DNA binding affinity and sequence selectivity of small molecules. Bioorg Med Chem 9:2511-2518 [PubMed: 11553493]

18. German N, Malik M, Rosen JD, Drlica K, Kerns RJ (2008) Use of gyrase resistance mutants to guide selection of 8-methoxy-quinazoline-2,4-diones. Antimicrob Agents Chemother. 52:39153921 [PubMed: 18765690]

19. NCI-60 Screening Methodology. https://dtp.cancer.gov/discovery_development/nci-60/ methodology.htm. Accessed August 2, 2018

20. Treatment of Colon Cancer, by Stage. https://www.cancer.org/cancer/colon-rectal-cancer/ treating/by-stage-colon.html Accessed August 2, 2018 

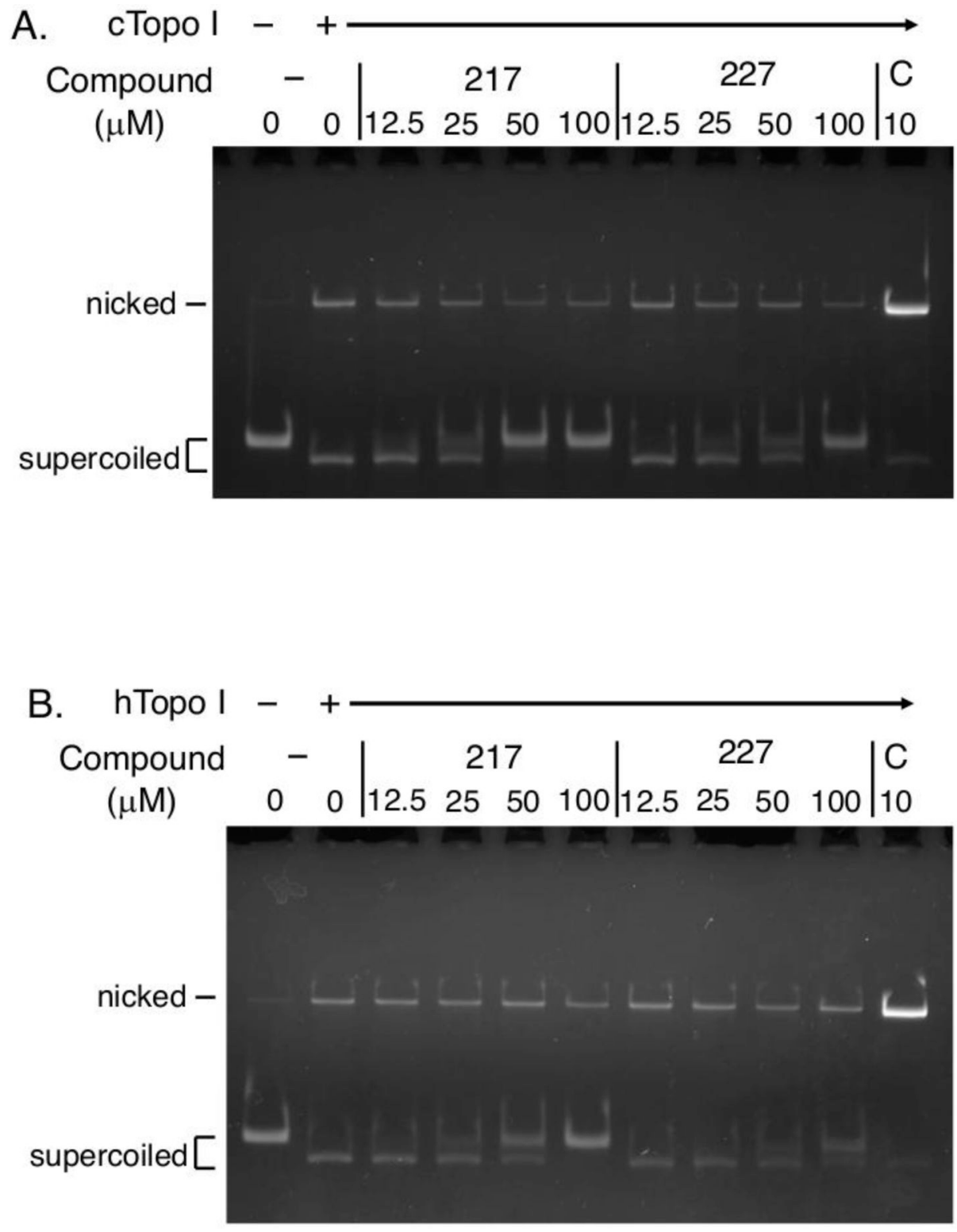

Figure 1. 217 and 227 do not poison either cTopo I or hTopo I.

The DNA cleavage assays were performed with either cTopo I (panel A) or hTopo I (panel B). Assays were repeated at least three times and essentially identical results were obtained. Representative results are shown here. Camptothecin $(C)$ was used as a control topoisomerase I poison. 


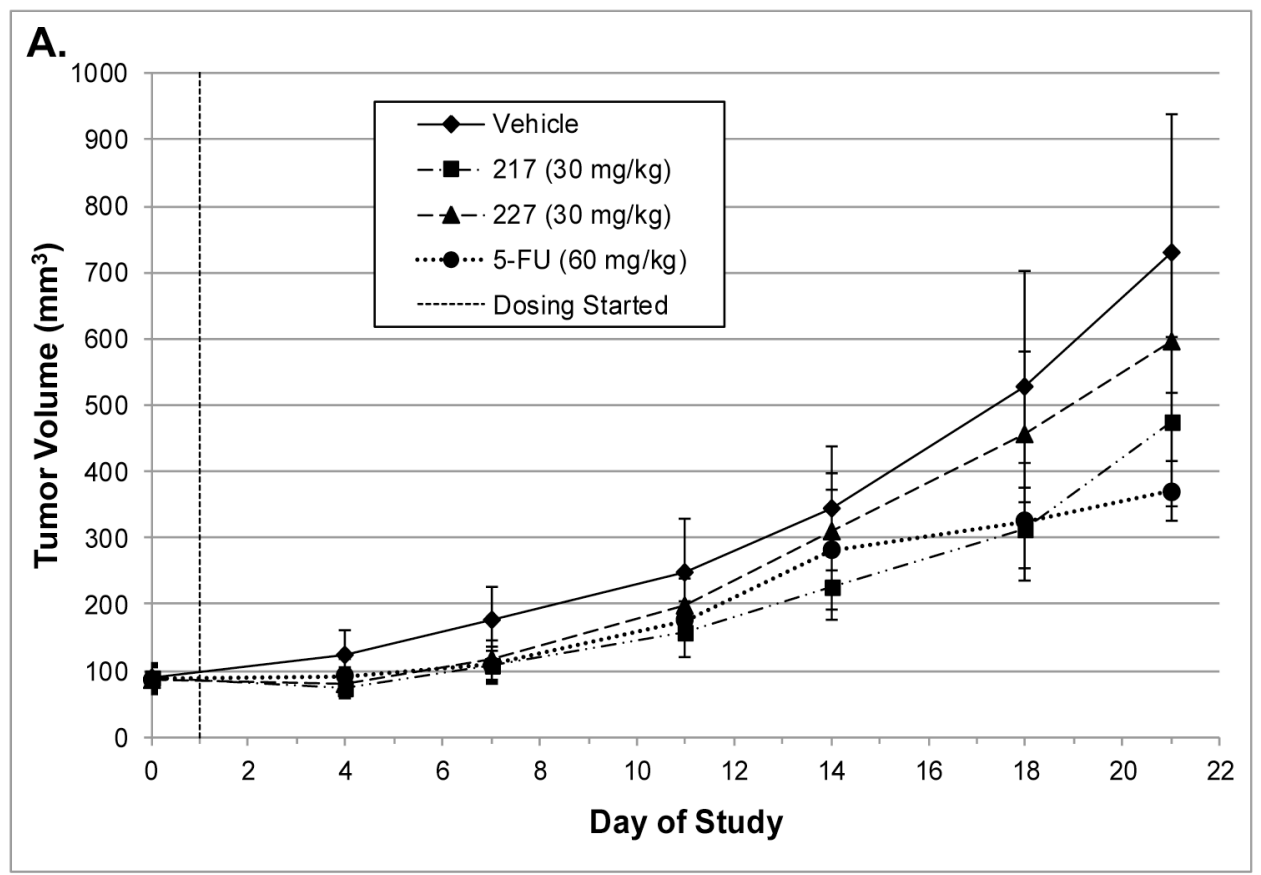

B.

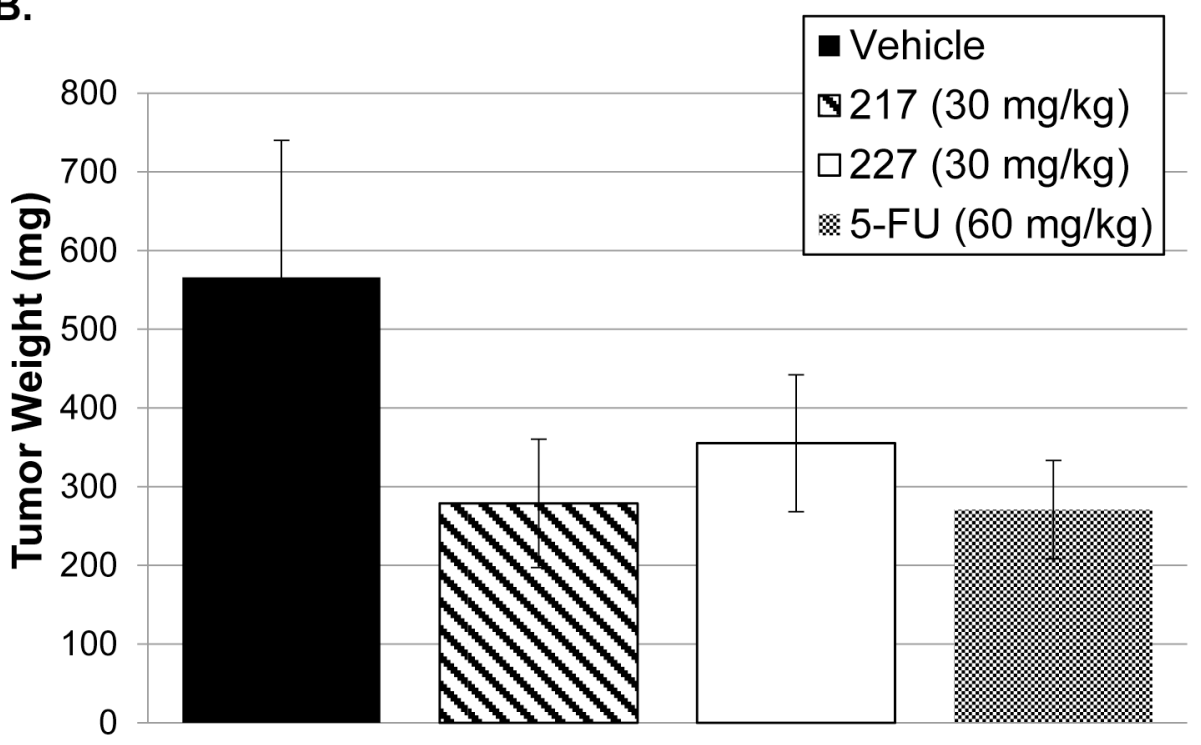

Figure 2. 217 and 227 can suppress the proliferation of colon cancer in vivo.

The average tumor volume (panel A) in the HT-29 colon cancer xenograft model was measured during a 3-week treatment with the daily, IP administration of 217 and 227 at 30 $\mathrm{mg} / \mathrm{kg}$. Tumor weight (panel B) was measured after the 3-week treatment. Ten mice were used in each treatment group. 
Table 1.

Activities 217 and 227 against type I topoisomerases ${ }^{1}$

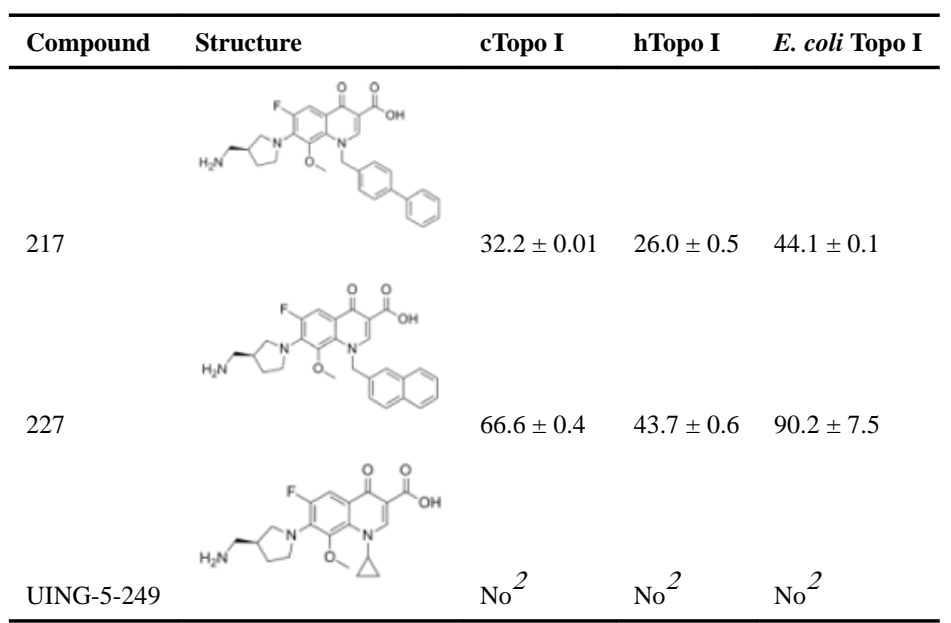

${ }^{1}$ The IC50 values (the 50\% inhibitory concentration, $\mu \mathrm{M}$ ) of 217 and 227 against calf, human, and $E$. coli Topo Is were determined in the relaxation assays.

2 No, no inhibition was detected when the relaxation assays were performed in the presence of $100 \mu \mathrm{M}$ UING-5-249 (18), a control fluoroquinolone. 
Table 2.

In vitro anti-proliferative activities of 217 and $227^{1}$

\begin{tabular}{lll}
\hline Compound & $\mathbf{G I}_{\mathbf{5 0}}(\boldsymbol{\mu M})$ & $\mathbf{L D}_{\mathbf{5 0}}(\boldsymbol{\mu M})$ \\
\hline 217 & 1.9 & 36.3 \\
227 & 3.1 & 70.8 \\
\hline
\end{tabular}

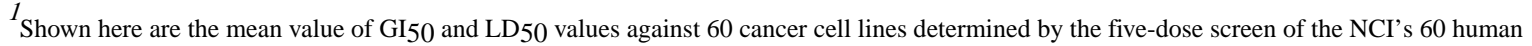
cell lines screening (19). 